\title{
miR106b regulates retinoblastoma Y79 cells through Runx3
}

\author{
GE YANG ${ }^{1}$, YANG FU ${ }^{2}$, LUXI ZHANG ${ }^{1}$, XIAOYAN LU $^{1}$ and QIUMING LI ${ }^{1}$ \\ Departments of ${ }^{1}$ Ophthalmology and ${ }^{2}$ General Surgery, The First Affiliated Hospital of Zhengzhou University, \\ Zhengzhou, Henan 450052, P.R. China
}

Received February 20, 2017; Accepted July 21, 2017

DOI: $10.3892 /$ or.2017.5931

\begin{abstract}
MicroRNAs are increasingly recognized as important regulators of cancer. The aim of the present study was to investigate the role of miR-106b in the regulation of Y79 retinoblastoma. Y79 cells were transfected with antisense oligonucleotides (ASO) against miR-106b (ASO-miR-106b) or ASO-control. After transfection, the levels of miR-106b were monitored with real-time PCR (RT-PCR). The effects of ASO-miR-106b transfection on cell viability was evaluated by Cell Counting Kit-8 (CCK-8) analysis at 24, 48 and $72 \mathrm{~h}$ after transfection. Subsequently, the cells were stained with Annexin V-FITC and propidium iodide (PI) and subjected to flow cytometry to assess cell apoptosis. Transwell assay was used to analyze cell migration. Changes in Runt-related transcription factor 3 (Runx3) mRNA and proteins levels were also evaluated. miR-106b was downregulated by ASO-miR-106b at 48 and $72 \mathrm{~h}$ after transfection, accompanied by a decrease in cell viability and proliferation, as well as an increase in cell apoptosis. Transwell analysis indicated that cells treated with ASO-miR-106b exhibited significantly lower cell migratory abilities. The mRNA and protein level of Runx3 were upregulated after transfection. These results demonstrated that suppression of miR-106b inhibited Y79 cell proliferation and migration. The upregulation of Runx3 after miR-106b suppression ascertained that Runx 3 is a tumor-suppressor in retinoblastoma and is a target of $\mathrm{miR}-106 \mathrm{~b}$.
\end{abstract}

\section{Introduction}

Retinoblastoma is an aggressive eye cancer and the most common intraocular cancer of infancy and childhood (1). Previous studies have indicated that various vulnerable groups, such as earthquake survivors (2) and rural-tourban migrants (3), were are in poor health, suffer from retinoblastoma. In addition, studies on retinoblastoma have

Correspondence to: Dr Qiuming Li, Department of Ophthalmology, The First Affiliated Hospital of Zhengzhou University, 1 Jianshe East Road, Zhengzhou, Henan 450052, P.R. China

E-mail: qiumingli111@126.com

Key words: miR-106b, retinoblastoma, Runx3, Y79, antisense oligonucleotides led to the discovery of the first tumor-suppressor gene, $R B 1$, the loss of which accounts for aberrant cell cycle arrest and the tumorigenesis of retinoblastoma. Despite research into the $R B$ family and cell cycle control, the specific pathways that contribute to the $R B$ loss in tumorigenesis remain largely unknown. In addition, mice with $R B$ deletion alone were not retinoblastoma-prone (4), suggesting the existence of other mechanisms that cooperatively contributes to the initiation of retinoblastoma.

It is increasingly recognized that microRNAs (miRNAs) are important regulators of cancer. They are short non-coding RNAs that post-transcriptionally modulate the expression of cancer-related genes (5). In retinoblastoma, a number of miRNAs have been identified to synergize with loss of the $R B$ family to regulate cell cycles (6-8). Besides the $R B$ family, the Runt-related transcription factor 3 (Runx3) gene, another tumor suppressor in retinoblastoma, was also found to be regulated by miRNAs. Runx3 is located on the chromosome $1 \mathrm{p} 36$ and plays an important role in mammalian development (9). The loss of Runx 3 has been considered as a prognostic marker for bladder tumor (10), gastric cancer (11) and glioblastoma (12). Previously, the link between miR-106b and Runx3 has been demonstrated in laryngeal carcinoma (13). By binding to the 3'-untranslated region (3'-UTR) of Runx3, miR-106b downregulated Runx3 expression, which consequently abolished the proliferation and invasion of laryngeal carcinoma cells (13). In retinoblastoma, although the downregulation of Runx3 has been documented, the mechanism involved in the regulation of Runx3 has not been investigated.

Surgery remains the primary treatment for retinoblastoma to date (14). However, being an invasive procedure, surgery exposes patients to substantial risk of losing vision (1). Chemotherapy has also been exploited for retinoblastoma, however chemotherapy is associated with potential toxicity and it also increases the risk of secondary cancers $(15,16)$. Therefore, strategies capable of modulating retinoblastoma-causative genes are urgently needed to improve the clinical outcome of retinoblastoma therapy and minimize harm to the eye.

In the present study, we strived to elucidate the role of miR-106b in retinoblastoma and investigated the correlation between miR-106b and Runx3. We revealed that inhibition of miR-106b led to decreased cell proliferation and migration of human Y79 retinoblastoma cells. Concurrently, upregulation of Runx3 was observed. The results shed light on the target of miR-106b in retinoblastoma, and provide a novel strategy for inhibiting retinoblastoma progression. 


\section{Materials and methods}

Cell culture and transfection. Human retinoblastoma Y79 cells were acquired from the American Type Culture Collection (ATCC; Rockville, MD, USA), and were maintained by Beina Co. Ltd. (Beijing, China). Dulbecco's modified Eagle's medium (DMEM; Invitrogen, Carlsbad, CA, USA) containing 5\% fetal bovine serum (FBS; HyClone, Logan, UT, USA), $2.0 \mathrm{~g} / 1$ sodium bicarbonate, $1 \times 10^{5} \mathrm{IU} / 1$ penicillin and $100 \mathrm{mg} / 1$ streptomycin were used for cell culture. The cells were cultured in an incubator maintained at $37^{\circ} \mathrm{C}$ with saturated humidity and $5 \% \mathrm{CO}_{2}$. ASO-miR-106b or ASO-control of $50 \mathrm{nM}$ was used for transfection with Lipofectamine ${ }^{\circledR} 2000$ (Invitrogen) according to the manufacturer's recommendations. Briefly, cells were seeded into 6-well plates at a density of $10^{5}$ cells/well and cultured overnight. In each well, $100 \mathrm{pmol}$ of ASO-control or ASO-miR-106b was added along with $5 \mu \mathrm{l}$ Lipofectamine ${ }^{\circledR}$ 2000. ASO-miR-106b and ASO-control were prepared by Jiman Phamaceuticals (Shanghai, China).

Cell viability assay. For the cell viability assay, cells of 2.5-3 $\times 10^{4}$ cells/well were first seeded into 96-well plates, with each well containing $100 \mu \mathrm{l}$ medium, and treated with ASO-miR-106b or ASO-control. After culturing for 24, 48 and 72 h, $10 \mu \mathrm{l}$ Cell Counting Kit-8 (CCK-8) solution was added into the medium, and incubated for another 4-5 h. The absorbance of each well at $538 \mathrm{~nm}$ was assessed to calculate the cell viability using the following equation:

$$
\text { Cell viability }=\frac{O D_{\text {untreated }}-O D_{\text {treated }}}{O D_{\text {untreated }}}
$$

Transwell cell migration assay. The Transwell apparatus was coated with Matrigel (both from BD Biosciences, San Jose, CA, USA) of 20-30 $\mu \mathrm{l}$ and allowed to gel overnight at $37^{\circ} \mathrm{C}$. The other side of the membrane was coated with fibronectin (Invitrogen). Y79 cells ( $5 \times 10^{5}$ cells $\left./ \mathrm{ml}\right)$ of $2,000 \mu 1$ were added to the chamber and cultured for $24 \mathrm{~h}$ before removing the cells on the membrane. The membrane on the lower chamber was collected and fixed with formalin for $30 \mathrm{~min}$, and stained with hematoxylin. Dehydration was carried out using the standard procedure using ethanol. The membrane, which was dehydrated with ethanol and xylene, was mounted onto cover slips, followed by cell counting in 4 fields of view. The average cell number was calculated.

Apoptosis analysis using flow cytometry. Cells collected at 48 and $72 \mathrm{~h}$ after transfection were washed with $0.01 \mathrm{~mol} / \mathrm{l}$ phosphate-buffered saline (PBS), and centrifuged at $1,500 \mathrm{rpm} / \mathrm{m}$ for $5 \mathrm{~min}$. The supernatant was then discarded. Cell pellets were re-suspected to ensure a cell density of $1 \times 10^{6}$ cells $/ \mathrm{ml}$. A cell suspension of $500 \mu 1$ was then dispensed into a microcentrifuge tube, followed by the addition of $5 \mu 1$ Annexin V-FITC and $10 \mu \mathrm{l}$ propidium iodide (PI) (Lianke Biology Co., Ltd., Hangzhou, China) for staining for $10 \mathrm{~min}$ at room temperature. Flow cytometry was used to detect cells with apoptotic activity. Experiments were performed in triplicate for each sample.
$R T-P C R$. Total RNA of the sample was extracted using a RNA Extract kit (Promega, Madison, WI, USA). Synthesis of cDNA was performed using $1 \mu \mathrm{g}$ purified RNA and a kit acquired from Takara (Shiga, Japan). RT-PCR was carried out using SYBR-Green mixture (Takara). Quantification was performed using the $2^{-\Delta \Delta \mathrm{Ct}}$ method. Primers used in the present study were as follows: 5'-GGATTTGGTCGTATTGGGCG-3' (sense) and 5'-TACTTCTCATGGTTCACAC-3' (antisense) for GAPDH; 5'-TGCGGCAACACCAGTCGATGG-3' (sense) and 5'-CCA GTGCAGGGTCCGAGGT-3' (antisense) for miR-106b; 5'-TGGCAGGCAATGACGA-3' (sense) and 5'-CAGGGAACG GCTTGGT-3' (antisense) for Runx3. Primers were synthesized by Sangon Biotech (Shanghai, China).

Western blot analysis. Cells transfected for 48 and $72 \mathrm{~h}$ were lyzed using RIPA cell lysis buffer (Thermo Fisher Scientific, Inc., Waltham, MA, USA). The protein concentration was determined using the BCA assay (Pierce, Rockford, IL, USA). SDS-PAGE was performed using $40 \mu \mathrm{g}$ of protein. The protein was then transferred onto the polyvinylidene difluoride (PVDF) membrane, followed by blocking with $50 \mathrm{~g} / 1$ non-fat milk for $1.5 \mathrm{~h}$. The goat anti-human Runx3 antibody (Santa Cruz Biotechnology, Santa Cruz, CA, USA) was diluted (1:500) in $1 \% \mathrm{BSA}$ and incubated with the membrane at $4^{\circ} \mathrm{C}$ overnight. After washing with TBST (1\% Tween-20) 3 times (6 min each time), an HRP-conjugated anti-goat antibody (1:5,000 dilution; Boster Biotechnology Co. Ltd., Wuhan, China) was applied to the membrane and was incubated for $2 \mathrm{~h}$. Visualization of the protein band was performed by adding ECL substrates in the dark. The band intensities of GAPDH were used to normalize the expression of the Runx 3 protein.

Statistical analysis. Statistical analysis was performed using SPSS 19.0 software package (IBM, Chicago, IL, USA). All data were presented as the mean \pm SD. Differences were considered significant when $\mathrm{P}<0.05$.

\section{Results}

Transfection of ASO-miR-106b downregulates miR-106b expression. To validate the efficacy of ASO-miR-106b in downregulating miR-106b expression, RT-PCR was performed in Y79 cells transfected with ASO-miR-106b or ASO-control. As shown in Fig. 1, at $24 \mathrm{~h}$ after transfection, no clear difference in miR-106b levels was seen among all groups. After 48 and $72 \mathrm{~h}$, a significant downregulation of miR-106b was observed in cells transfected with miR-106b $(\mathrm{P}<0.01)$, suggesting that ASO-miR-106 effectively inhibited the expression of miR-106. Conversely, no significant differences were observed in cells transfected with the ASO-control $(\mathrm{P}>0.05)$.

Downregulation of miR-106b decreases cell viability. Concomitant with the inhibition of miR-106b expression, the viability of Y79 was also decreased. As shown in Fig. 2, while no significant viability suppression was seen at $24 \mathrm{~h}$ after transfection, at 48 and $72 \mathrm{~h}$ after transfection, cell viability was significantly decreased, as revealed by CCK- 8 assay $(\mathrm{P}<0.05)$.

Downregulation of miR-106b decreases cell migration. We next examined whether downregulation of miR-106b decreased 


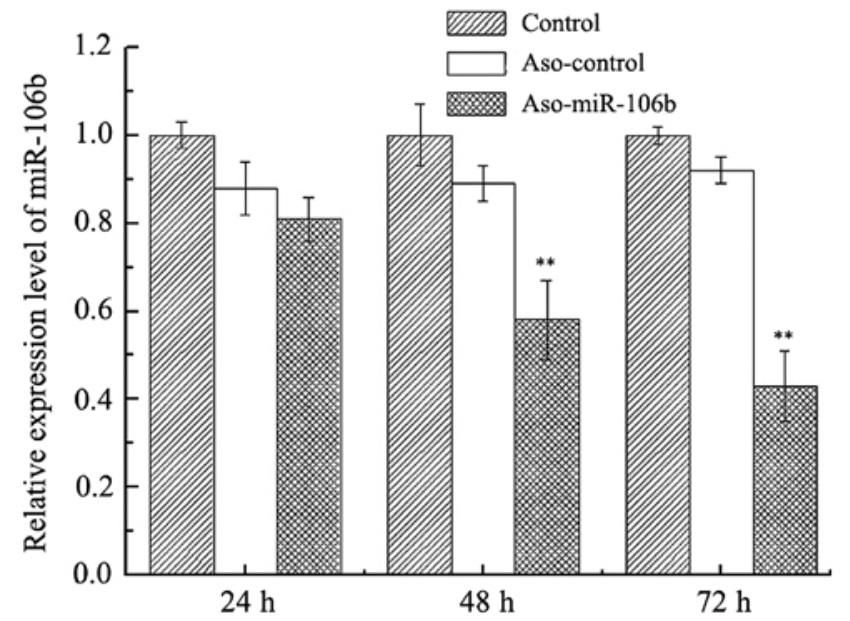

Figure 1. Relative expression level of miR-106b in Y79 cells transfected with ASO-control or ASO-miR-106b at 24, 48 and $72 \mathrm{~h}$. The levels of miR-106b in Y79 cells without transfection were used for normalization. Compared with control group; ${ }^{* *} \mathrm{P}<0.01$.

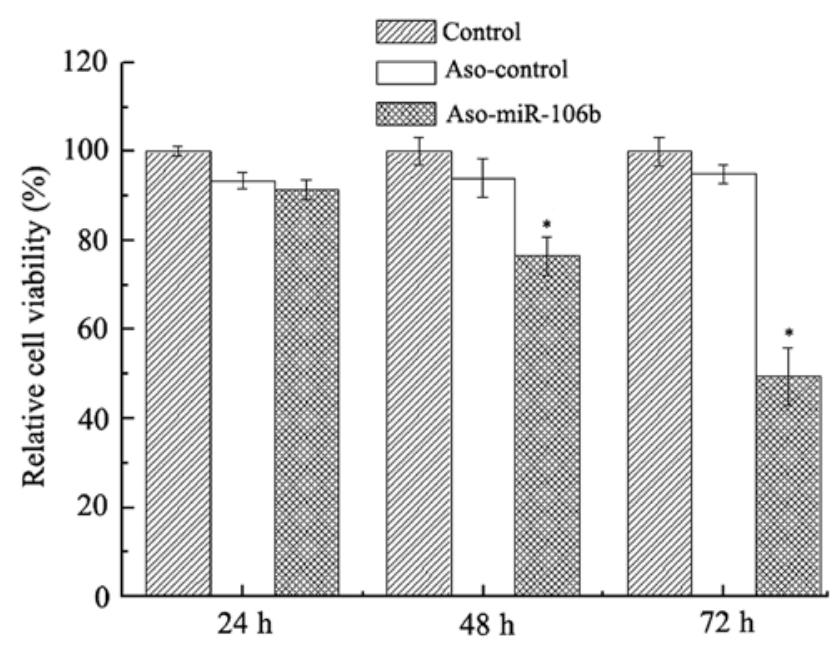

Figure 2. Cell viability analysis in cells transfected with ASO-control or ASO-miR 106 at $24 \mathrm{~h}, 48 \mathrm{~h}$, and $72 \mathrm{~h}$ after transfection, as revealed by CCK- 8 assay. Compared with control group; ${ }^{*} \mathrm{P}<0.05$.

the migration of Y79 cells. As expected, at 48 and $72 \mathrm{~h}$ after transfection, the migration of Y79 cells was significantly decreased as indicated by Transwell assay $(\mathrm{P}<0.05)$ (Fig. 3). In contrast, transfection with ASO-control did not suppress cell migration.

Apoptosis is increased with miR-106 downregulation. After transfection for 48 and $72 \mathrm{~h}$, the apoptosis in Y79 cells was evaluated with Annexin-PI staining and flow cytometry. Consistent with the decreased cell viability and migration in Y79 cells after ASO-miR-106b transfection, a shift of cell population toward the Annex ${ }^{+}-\mathrm{PI}^{+}$population was recorded (Figs. 4 and 5), indicating an increase in apoptotic activity.

Downregulation of miR-106b increases Runx3 expression. To explore the target of miR-106b in retinoblastoma, we analyzed the expression level of Runx $3 \mathrm{mRNA}$ and protein in Y79 cells after ASO-miR-106b transfection. The results revealed that both Runx3 mRNA and protein expression

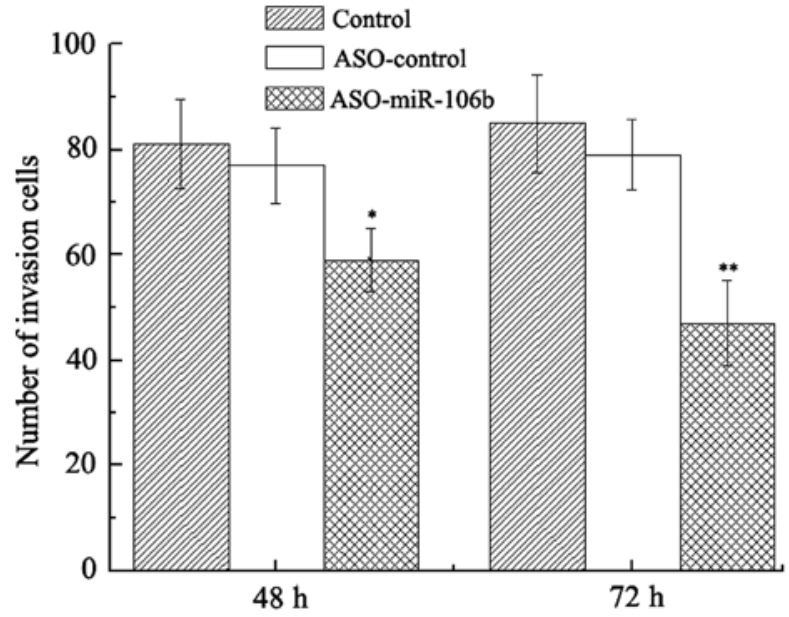

Figure 3. The number of invaded cells as determined by Transwell assay in Y79 cells transfected with ASO-control or ASO-miR-106b. Cells that received no transfection were used as a control. Compared with control group; ${ }^{*} \mathrm{P}<0.05 ;{ }^{* *} \mathrm{P}<0.01$.

levels were upregulated after ASO-miR-106b transfection $(\mathrm{P}<0.05)$ (Figs. 6 and 7). Given the role of Runx3 as a tumor suppressor, this Runx3 upregulation after miR-106b inhibition is consistent with the diminished cell proliferation and migration. Therefore, Runx 3 acts as a target of miR-106b in the regulation of retinoblastoma.

\section{Discussion}

A vast majority of people suffer from cancer $(17,18)$. By fine-tuning the expression of multiple genes post-transcriptionally, miRNAs offer a new paradigm for re-modulating the gene network wired to the thriving cancer (19). Numerous miRNA-based strategies for correcting aberrant gene expression in cancer have emerged. These miRNAs may assume tumor-promoting or tumor-inhibiting roles in cancer. For example, given that the loss of $R B 1$ expression is a hallmark of cancer, miR-106a inhibition was employed to reverse the downregulation of $R B I$ (20). In another study, miR-192 was ectopically overexpressed, which inhibited cell proliferation and induced cell apoptosis in lung cancer cells (21). For retinoblastoma, recent miRNA microarray analysis yielded a panel of miRNAs as essential effectors that modulate cancer progression, metastasis and resistance (22). Nevertheless, these miRNAs have yet to be utilized as therapeutic targets in retinoblastoma. Furthermore, despite extensive studies on the etiology of the disease and genetic diagnosis approaches (23), gene therapies for retinoblastoma are rarely studied. Therefore, in the present study, we set forth to explore the therapeutic value of miR-106b in retinoblastoma. The present study is preceded by much effort in correlating miR-106b overexpression to the malignant phenotype and resistance of cancer $(24,25)$. We demonstrated that the inhibition of miR-106b induced a decrease in Y79 retinoblastoma cell viability and migration, and induced cell apoptosis. Instead of directly targeting oncogenes of retinoblastoma, our approach was to inhibit the expression of miR-106b with antisense oligonucleotides (ASO) to exert the antitumor effects. Our data potentiated the development of an in vivo gene therapy strategy that targets 


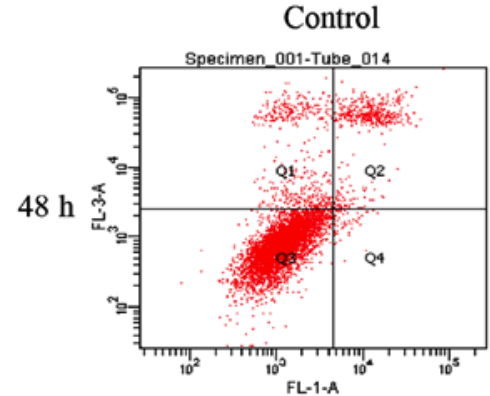

Control

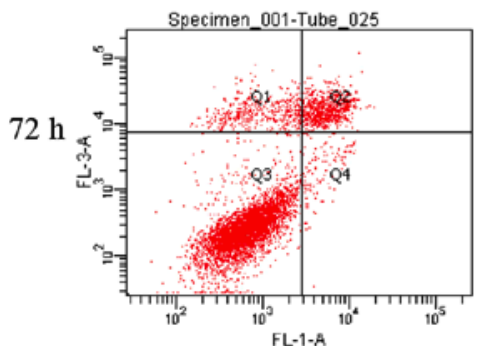

ASO-control

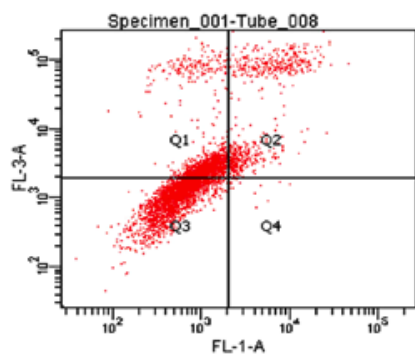

ASO-control

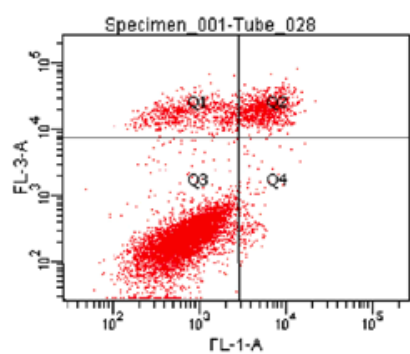

ASO-miR-106b

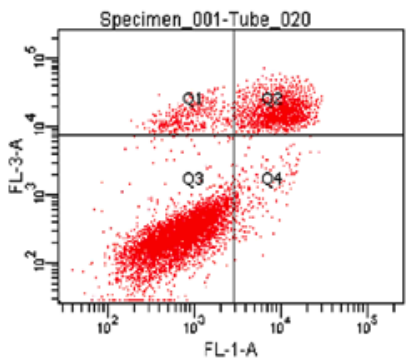

ASO-miR-106b

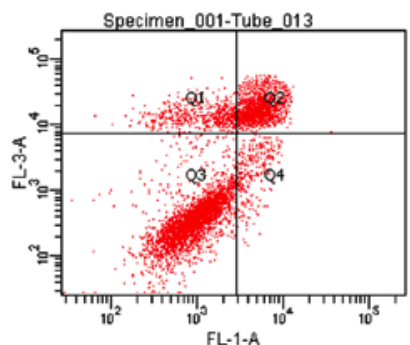

Figure 4. Apoptosis analysis with Annexin V/PI staining and flow cytometry in Y79 cells transfected with ASO-miR-106b or ASO-control. Y79 cells that received no transfection were used as a control.

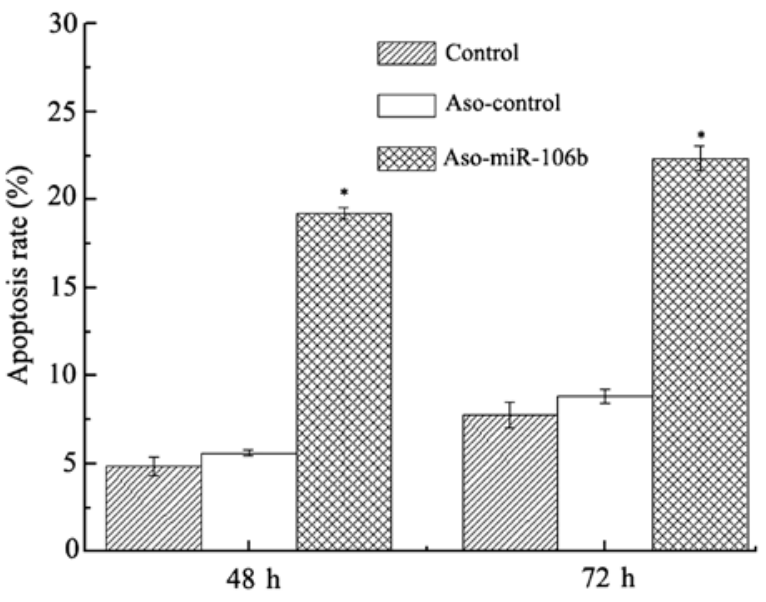

Figure 5. Apoptotic rates in Y79 cells. Y79 cells transfected with ASOcontrol, or Y79 cells transfected with ASO-miR-106b, as indicated by flow-cytometry as shown in Figure 4. Compared with the control group; ${ }^{*} \mathrm{P}<0.05$.

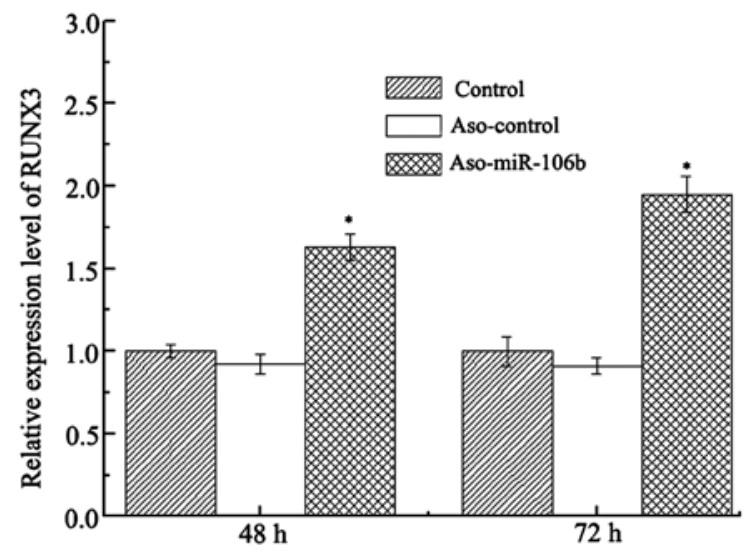

Figure 6. Runx3 mRNA levels in Y79 cells without transfection, or cells transfected with ASO-control or ASO-miR-106b. Compared with control group; ${ }^{*} \mathrm{P}<0.05$.

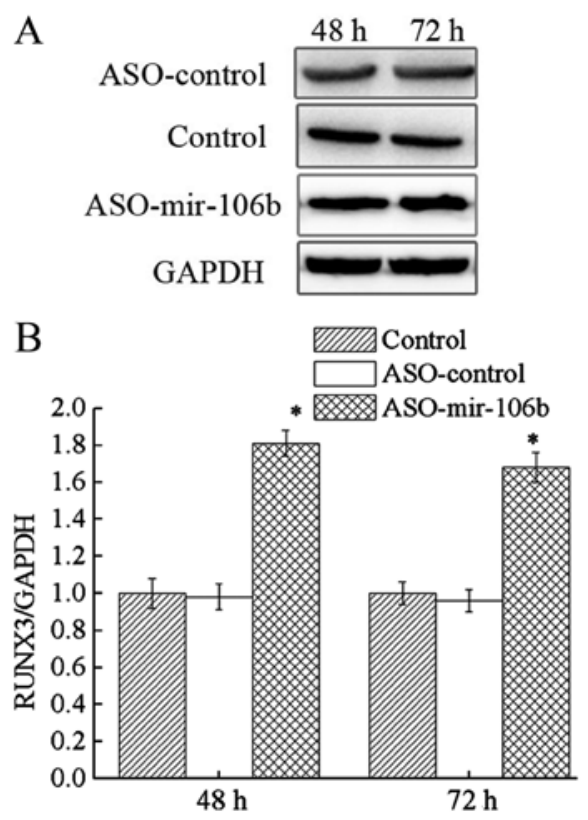

Figure 7. (A) Western blot analysis of Runx3 levels in Y79 cells that received no transfection (ASO-control transfection), or ASO-miR-106b transfection. GAPDH was used as a loading control. (B) Quantification of Runx3 protein levels in Y79 cells transfected with ASO-control or ASO-miR-106b. Compared with control group; ${ }^{\mathrm{P}}<0.05$.

miR-106b; a strategy that lowers the non-specific toxicity commonly observed with chemotherapy drugs. With the advances in RNA-delivery systems, we could envision that retinoblastoma therapy based on miR-106b inhibition may improve the clinical outcome of patients and exempt them from painful and risky surgery.

Furthermore, we revealed that the antitumor effect of anti-miR-106b therapy was mediated by Runx3. Similar to $R B 1$, Runx 3 serves as a tumor suppressor and its methylation is pivotal to the transforming growth factor- $\beta$ (TGF- $\beta$ ) pathway. 
Since its link to epithelial-to-mesenchymal transition and cancer metastasis, the loss of Runx3 significantly affects the clinical outcome of cancer patients (26). In spite of efforts in unraveling the role of Runx 3 in cancer progression, very few Runx3-based therapeutic strategies have been devised. Previously, ectopic expression of Runx3 was exploited as an antitumor strategy, whereby the restoration of Runx3 was shown to drastically suppress tumor growth (26). The findings here revealed that inhibition of miR-106b is another viable avenue to upregulate Runx3. This mirrors recent evidence that revealed that miR-106b significantly promotes TGF- $\beta$ signaling and cancer metastasis $(25,27)$. However, the fact that miR-106b is also an important player in the PTEN/PIK3/AKT pathway (27), WNT pathway (28) and RB family enhances its clinical value as a target in cancer.

In summary, we demonstrated that anti-miR-106b therapy effectively inhibited Y79 retinoblastoma cell viability, proliferation and induced cell apoptosis in vitro. Runx3 was found to be a target of miR-106b, and the inhibition of miR-106b upregulated Runx3. Our data is significant for the development of novel strategies in gene therapy for retinoblastoma. Further in vivo studies are warranted to corroborate the role of miR$106 \mathrm{~b}$ in retinoblastoma, and potentially apply this strategy to the clinic.

\section{Acknowledgements}

The present study received no specific grant from any funding agency in the public, commercial or non-profit sectors.

\section{References}

1. Dimaras H, Kimani K, Dimba EA, Gronsdahl P, White A, Chan HS and Gallie BL: Retinoblastoma. Lancet 379: 1436-1446, 2012.

2. Liang Y and Cao R: Employment assistance policies of Chinese government play positive roles! The impact of post-earthquake employment assistance policies on the health-related quality of life of Chinese earthquake populations. Soc Indic Res 120: 835-857, 2014.

3. Liang Y and Guo M: Utilization of health services and health-related quality of life research of rural-to-urban migrants in china: A cross-sectional analysis. Soc Indic Res 120: 277-295, 2015.

4. Clarke AR, Maandag ER, van Roon M, van der Lugt NM, van der Valk M, Hooper ML, Berns A and te Riele H: Requirement for a functional Rb-1 gene in murine development. Nature 359: 328-330, 1992

5. Lu J, Getz G, Miska EA, Alvarez-Saavedra E, Lamb J, Peck D, Sweet-Cordero A, Ebert BL, Mak RH, Ferrando AA, et al: MicroRNA expression profiles classify human cancers. Nature 435: 834-838, 2005.

6. Conkrite K, Sundby M, Mukai S, Thomson JM, Mu D, Hammond SM and MacPherson D: miR-17 92 cooperates with RB pathway mutations to promote retinoblastoma. Genes Dev 25: 1734-1745, 2011

7. Xu Y, Wang K, Gao W, Zhang C, Huang F, Wen S and Wang B: MicroRNA-106b regulates the tumor suppressor RUNX3 in laryngeal carcinoma cells. FEBS Lett 587: 3166-3174, 2013.

8. Trompeter HI, Abbad H, Iwaniuk KM, Hafner M, Renwick N, Tuschl T, Schira J, Müller HW and Wernet P: MicroRNAs MiR-17, MiR-20a, and MiR-106b act in concert to modulate E2F activity on cell cycle arrest during neuronal lineage differentiation of USSC. PLoS One 6: e16138, 2011.

9. Tucker A and Sharpe P: The cutting-edge of mammalian development; how the embryo makes teeth. Nat Rev Genet 5: 499-508, 2004.
10. Kim EJ, Kim YJ, Jeong P, Ha YS, Bae SC and Kim WJ: Methylation of the RUNX3 promoter as a potential prognostic marker for bladder tumor. J Urol 180: 1141-1145, 2008.

11. Guo WH, Weng LQ, Ito K, Chen LF, Nakanishi H, Tatematsu M and Ito Y: Inhibition of growth of mouse gastric cancer cells by Runx3, a novel tumor suppressor. Oncogene 21: 8351-8355, 2002.

12. Mueller W, Nutt CL, Ehrich M, Riemenschneider MJ, von Deimling A, van den Boom D and Louis DN: Downregulation of $R U N X 3$ and TES by hypermethylation in glioblastoma. Oncogene 26: 583-593, 2007.

13. Honavar SG, Shields CL, Shields JA, Demirci H and Naduvilath TJ: Intraocular surgery after treatment of retinoblastoma. Arch Ophthalmol 119: 1613-1621, 2001.

14. Errico A: Cancer therapy: Retinoblastoma - chemotherapy increases the risk of secondary cancer. Nat Rev Clin Oncol 11: 623-623, 2014.

15. Smith SJ and Smith BD: Evaluating the risk of extraocular tumour spread following intravitreal injection therapy for retinoblastoma: A systematic review. Br J Ophthalmol 97: 1231-1236, 2013.

16. Tong AW and Nemunaitis J: Modulation of miRNA activity in human cancer: A new paradigm for cancer gene therapy? Cancer Gene Ther 15: 341-355, 2008 .

17. Liang Y: Satisfaction with economic and social rights and quality of life in a post-disaster zone in China: Evidence from earthquake-prone Sichuan. Disaster Med Public Health Prep 9: 111-118, 2015.

18. Liang Y: Correlations between health-related quality of life and interpersonal trust: Comparisons between two generations of Chinese rural-to-urban migrants. Soc Indic Res 123: 677-700, 2015.

19. Volinia S, Calin GA, Liu CG, Ambs S, Cimmino A, Petrocca F, Visone R, Iorio M, Roldo C, Ferracin M, et al: A microRNA expression signature of human solid tumors defines cancer gene targets. Proc Natl Acad Sci USA 103: 2257-2261, 2006.

20. Feng S, Cong S, Zhang X, Bao X, Wang W, Li H, Wang Z, Wang G, Xu J, Du B, et al: MicroRNA-192 targeting retinoblastoma 1 inhibits cell proliferation and induces cell apoptosis in lung cancer cells. Nucleic Acids Res 39: 6669-6678, 2011.

21. Zhao JJ, Yang J, Lin J, Yao N, Zhu Y, Zheng J, Xu J, Cheng JQ, Lin JY and Ma X: Identification of miRNAs associated with tumorigenesis of retinoblastoma by miRNA microarray analysis. Childs Nerv Syst 25: 13-20, 2009.

22. Zhang J, Benavente CA, McEvoy J, Flores-Otero J, Ding L, Chen X, Ulyanov A, Wu G, Wilson M, Wang J, et al: A novel retinoblastoma therapy from genomic and epigenetic analyses. Nature 481: 329-334, 2012.

23. Zheng L, Zhang Y, Liu Y, Zhou M, Lu Y, Yuan L, Zhang C, Hong M, Wang S and Li X: MiR-106b induces cell radioresistance via the PTEN/PI3K/AKT pathways and p21 in colorectal cancer. J Transl Med 13: 252, 2015.

24. Gong C, Qu S, Liu B, Pan S, Jiao Y, Nie Y, Su F, Liu Q and Song E: MiR-106b expression determines the proliferation paradox of TGF- $\beta$ in breast cancer cells. Oncogene 34: 84-93, 2015.

25. Wei D, Gong W, Oh SC, Li Q, Kim WD, Wang L, Le X, Yao J, Wu TT, Huang S, et al: Loss of RUNX3 expression significantly affects the clinical outcome of gastric cancer patients and its restoration causes drastic suppression of tumor growth and metastasis. Cancer Res 65: 4809-4816, 2005

26. Smith AL, Iwanaga R, Drasin DJ, Micalizzi DS, Vartuli RL and Ford HL: Abstract A20: The Six1-regulated miR-106b-25 cluster is a mediator of the tumor promotional effects of TGF- $\beta$ signaling in human breast cancer. Cancer Res 72 (Suppl 2): A20-A20, 2012.

27. Li YL, Wang YH, Zhou H, Yong YH and Cao YD: miR-106b promoted growth and inhibited apoptosis of nasopharyngeal carcinoma cells by suppressing the tumor suppressor PTEN. Int J Clin Exp Pathol 9: 7078-7086, 2016.

28. Malcomson FC, Willis ND, McCallum I, Xie L, Kelly S, Bradburn M, Belshaw NJ, Johnson IT and Mathers JC: Differences in the expression of microRNAs implicated in colorectal carcinogenesis and involved in the WNT signalling pathway in the macroscopically-normal epithelium of people at higher-risk of colorectal cancer. Proc Nutr Soc 74 (OCE1): E46, 2015. 